\title{
Is Disability a Health Problem?
}

\author{
Malcolm MacLachlan ${ }^{1,2, *}$ and Hasheem Mannan ${ }^{1,3}$ \\ ${ }^{1}$ Centre for Global Health \& School of Psychology, Trinity College Dublin, Dublin, Ireland; \\ E-Mail: mlachlan@tcd.ie (M.M.) \\ ${ }^{2}$ Centre for Rehabilitation Studies, Stellenbosch University, Stellenbosch, South Africa \\ ${ }^{3}$ Nossal Institute for Global Health, University of Melbourne, Victoria, Australia; \\ E-Mail: hmannan@unimelb.edu.au; Tel.: +61 390356184; Fax: +61 393476872 \\ * Corresponding author
}

Submitted: 27 November 2013 | In revised form: 16 December 2013 | Accepted: 20 December 2013 | Published: 27 December 2013

\begin{abstract}
We welcome Andrew Haig's critique of our paper, "Disability \& Health: A research agenda" [1] in Social Inclusion. Our paper sought to identify research priorities to better understand, provide enhanced services and a better quality of life for people with disabilities, particularly in relation to their health and wellbeing. Haig's [2] critique makes several important points that deserve serious consideration. His comments reflect a view of the relationship between disability and health which is different from the one we have espoused. Specifically, Haig argues that (a) disability is a health problem, (b) medical rehabilitation should be separated from Community Based Rehabilitation (CBR), and (c) the evidence base for medical rehabilitation is much stronger than for CBR. We address each of these points below arguing that while some types of disability clearly result from health problems; often disability is not experienced as a health problem; and sometimes, disability in interaction with restricted access is the cause of health problems.
\end{abstract}

Keywords: disability; health; social inclusion

\section{Causes and Effects}

The International Classification of Functioning, Disability and Health (ICF) acknowledges that there is no simple one-to-one correspondence between having a health condition (disease or disorder) and disability [3]. A health condition may or may not affect a person's body functions and structures, may or may not restrict their activity, and may or may not influence their participation in society. Furthermore environ- mental (e.g. accessible buildings) and personal (e.g. coping style) factors may mediate these relationships - determining how any relationship that may exist between health and disability is actually played out. Clearly, as illustrated in Haig's example of a person who is unable to walk due to a spinal cord injury, their activity limitation may meaningfully be attributed to a disorder (in this case an accident). Furthermore, their experience of using a wheel chair may also be associated with pressure sores, urinary tract infections, and 
so on. They will be grateful for the medical treatments that can relieve them of these symptoms and so improve their health and quality of life; these treatments may indeed be life-saving. In this example the cause of the person's 'problem' may be attributed to a health condition, and so may its consequences.

The cause of something and its consequences are not however necessarily of the same nature. Haig amusingly illustrates his argument by telling us that a 10 -years-old's failure to leap tall buildings in a single bound (re Superman movies) could be described as "dis-ability", but that it would be foolish and irrational to do so. We agree. Adding to this we would argue that not being able to leap tall buildings and having to stay home to do the washing up after dinner, are indeed quite different types of 'things'; even if in the fanciful mind of the 10-year old they are related.

The failure of diplomacy and the onset of bloody war are also different types of things, although sometimes they may be related. More pertinently, being born with a congenital limb 'deficiency'; being dyslexic; or having Downs Syndrome, may or may not be associated with health problems. Some people with these conditions will lead full and very healthy lives, rarely or perhaps never accessing services that could be described as 'medical rehabilitation'. Disability does not necessarily result from a health problem, any more than does being taller or shorter; having blue or brown eyes; having an IQ of 120 or 80 ; or being black or white. Without being overly prosaic human kind is hugely varied.

\section{Predisposing and Precipitating}

The latter comparison with disability may be informative in the context of health. Colour differences are associated with differences in some health conditions. For instance, in certain geographic areas people with dark skins have a relatively higher incidence of sickle cell anaemia [4]; yet we would not describe colour, ethnicity or 'race' as "health problems". Ethnicity may also be associated with practices that precipitate health problems; for instance, inadequate exposure to sunlight due to dress customs being associated with nutritional rickets [4]. Rather than 'treating' ethnic differences, we accept them, often cultivating tolerance of difference through pluralism [5].

None of this is to deny that many people with disabilities do have health problems (just as do many people without disabilities) or that for some people with disabilities their health problems are the cause of, concurrent with, or a consequence of, their disability: as we stated in our paper: "while disability is not a 'health problem', some people with disabilities do have increased health needs associated with their disability, and all people with disabilities have the same right to access health services as everyone else" ([1], p. 38). Having a health problem is however neither a necessary nor sufficient condition to have a disability, nor vice versa. We do not therefore agree that disability is "inexorably intertwined" with health, as Haig suggests.

It is also important to state the 'social model' view here-that it is not bodily or functional impairments which disable people, but rather the assumptions about how people are, which society enacts; limiting some people's activities and their participation, by creating barriers-physical, social and organisational. A society constructed around a normative view of 'ability' that excludes and limits some citizens will mean that they have less access to health promotion, health maintenance, illness prevention, and treatments for health conditions, and this exclusion may cause health problems [6], rather than their disability per se.

\section{Medical Rehabilitation}

Haig argues that medical rehabilitation should be separated from CBR and not relegated to a "vague" box within a multifaceted CBR matrix. The recently developed guidelines on CBR recognize that the rehabilitation process is not one-dimensional; rather they incorporate health, education, livelihood, social inclusion and empowerment components. Each of these components is divided into 5 further sub-components; constituting a matrix of 25 distinct but related areas [7]. For instance, the health component has subcomponents of promotion, prevention, medical care, rehabilitation and assistive devices. Haig characterises medical rehabilitation as having "intensively trained specialists, advanced facilities and expert coordination of a multidisciplinary team" and describes his own and obviously very valuable work in a "world class" treatment facility in the United States. Clearly this work is crucial and deserves to be well supported, but it is a leap over many tall buildings-and continents-from the experience of most people with disabilities; $80 \%$ of whom live in low or middle income countries [6] where such services are not a short- or even mediumterm prospect. This is not because of political or media priorities, as suggested by Haig, but rather because there is a moral and rational imperative to allocate scarce resources as equitably as possible, providing the greatest aggregate benefit to the largest possible number of people [8]. Yes, regrettably, this will mean that advanced facilities and intensively trained specialists will not be available to many who would undoubtedly benefit from them and who would love to be treated in "world class" centres.

We would not however support the separation of what Haig describes as medical rehabilitation services from other aspects of initiatives directed at promoting the rights to services and opportunities of people with disabilities. Successful rehabilitation does not end at the foot of the hospital bed; it is about enhancing capabilities, enabling participation and promoting inclusion in spaces and places where people live-in their community. To make this a reality requires integ- 
ration-not separation-of the different aspects of CBR. Medical rehabilitation is one component of a range of services and opportunities which can help people live better. Accepting this 'complimentary' rather than 'dominant' positioning of the crucial medical aspects of the process of rehabilitation requires some humility among specialists. Furthermore, because of the very fact of their being specialists, they may also need to recognise that they lack the purview to have a coordinating, indeed integrating, function across such a diverse domain.

As a very specific example, assistive technology is certainly relevant to the remit of medical rehabilitation, yet it extends far beyond it; incorporating psychosocial, engineering and human rights issues in high, medium and low-income country contexts $[9,10]$. Effective use of assistive technologies necessitates the integration, not separation, of activities across the broad range of components that may be relevant to the rehabilitation of those who use them.

\section{Evidence Based Practice}

Haig is clearly correct when he asserts that there is a stronger evidence base for rehabilitative medicine than for CBR, even after decades of practicing CBR. Work in CBR has often been inadequately evaluated and the optimum skills mixes and staff types needed to facilitate effective CBR are yet to be scientifically

\section{References}

1. Mannan $\mathrm{H}$, MacLachlan M. Disability and Health: A Research Agenda. Social Inclusion. 2013;1(1):3745.

2. Haig AJ. Disability Policy Must Espouse Medical as well as Social Rehabilitation. Social Inclusion. 2013;1(2):136-138.

3. World Health Organization Classification, Assessment, Surveys and Terminology Team. International Classification of Functioning, Disability and Health: Final Draft. Geneva, Switzerland: WHO; 2001. Available from: http://www.sustainable-design.ie/arc h/ICIDH-2Final.pdf (accessed on 27 November 2013).

4. MacLachlan M. Culture \& Health: A Critical Perspective towards Global Health. 2nd ed. Chichester, UK: Wiley; 2006.

5. MacLachlan M, O'Connell M, editors. Cultivating Pluralism: Cultural, Psychological and Social Perspectives on a Changing Ireland. Dublin, Ireland: Oak Tree Press; 2000.

6. World Health Organization, World Bank. World Report on Disability. Geneva, Switzerland: WHO; 2011. Available from: http://whqlibdoc.who.int/publica tions/2011/9789240685215_eng.pdf (accessed on 27 November 2013).

7. World Health Organization. Community-Based determined [11]. Haig's argument is recognition of the need for better monitoring, evaluation and research on CBR. The ethos of multi-disciplinary, cross-sectoral working is however in line with the Bamako Call for Health Research [12]; not addressing this challenge in CBR would be a negation of our responsibility-it is a call to action, entirely consistent with the research agenda we set out.

\section{Conclusion}

Our proposed research agenda is intended to encourage a more systematic and comparative approach to the relationship between disability and health. For sure, the inter-play between the two is, and will continue to be, contested. While we maintain that disability is not a health problem, we recognise that for some people it may well be associated with health problems, but also with human rights: indeed the 'oppositioning' of 'medical' and 'social' models, is not only unhelpful, but also unrealistic [13], and we support a view that recognises merits in each, and in combination, under some circumstances. We have also argued that the experiences that people with disabilities have of health services can be an excellent probe for evaluating the accessibility and effectiveness of health services in general; and in so doing disability can actually contribute to being a 'health problem solver', for all [14].

Rehabilitation: CBR Guidelines. Geneva, Switzerland: WHO; 2010.

8. Amin M, MacLachlan M, Mannan H, El Tayeb S, El Khatim A, Swartz L, Munthali A, Van Rooy G, McVeigh J, Eide A, Schneider M. EquiFrame: A Framework for Analysis of the Inclusion of Human Rights and Vulnerable Groups in Health Policies. Health \& Human Rights. 2011;13(2):1-20.

9. Desmond D, MacLachlan M. Psychosocial Issues in the Field of Prosthetics and Orthotics. Journal of Prosthetics \& Orthotics. 2002;12(2):12-24.

10. Borg J, Lindström A, Larsson S. Assistive technology in developing countries: National and international responsibilities to implement the Convention on the Rights of Persons with Disabilities. Lancet. 2009;374(9704):1863-1865.

11. MacLachlan M, Mannan H, McAuliffe E. Staff Skills not Staff Types for Community-Based Rehabilitation. Lancet. 2011;377(9782):1988-1989. doi:10.101 6/S0140-6736(10)61925-3.

12. The Lancet. The Bamako Call to Action: Research for Health. Lancet. 2008;372(9653):1855.

13. Shakespeare T. Disability Rights and Wrongs. Oxon, UK: Routledge; 2006.

14. MacLachlan M, Mannan H, McAuliffe E. Access to Health Care of Persons with Disabilities as an Indicator of Equity in Health Systems. Open Medicine. 2011;5(1):10-12. 